\title{
Infrared spectroscopy of hydrated sulfate dianions
}

\author{
Jia Zhou \\ Department of Chemistry, University of California, Berkeley, California 94720
}

Gabriele Santambrogio and Mathias Brümmer

Institut für Experimentalphysik, Freie Universität Berlin, Arnimallee 14, D-14195 Berlin, Germany

David T. Moore

Department of Chemistry, University of California, Berkeley, California 94720 and Chemical Sciences

Division, Lawrence Berkeley National Laboratory, Berkeley, California 94720

Ludger Wöste

Institut für Experimentalphysik, Freie Universitat Berlin, Arnimallee 14, D-14195 Berlin, Germany

\author{
Gerard Meijer \\ Fritz-Haber-Institut der Max-Planck-Gesellschaft, Faradayweg 4-6, D-14195 Berlin, Germany \\ Daniel M. Neumark ${ }^{\text {a) }}$ \\ Department of Chemistry, University of California, Berkeley, California 94720 and Chemical Sciences \\ Division, Lawrence Berkeley National Laboratory, Berkeley, California 94720
}

Knut R. Asmis ${ }^{\text {b) }}$

Institut für Experimentalphysik, Freie Universitat Berlin, Arnimallee 14, D-14195 Berlin, Germany and Fritz-Haber-Institut der Max-Planck-Gesellschaft, Faradayweg 4-6, D-14195 Berlin, Germany

(Received 4 August 2006; accepted 15 August 2006; published online 21 September 2006)

We report the first infrared spectra of multiply-charged anions in the gas phase. The spectra of $\mathrm{SO}_{4}^{2-} \cdot\left(\mathrm{H}_{2} \mathrm{O}\right)_{n}$, with $n=3-24$, show four main bands assigned to two vibrations of the dianionic core, the water bending mode, and solvent libration. The triply degenerate $\mathrm{SO}_{4}^{2-}$ antisymmetric stretch vibration probes the local solvent symmetry, while the solvent librational band is sensitive to the hydrogen bonding network. The spectra and accompanying electronic structure calculations indicate a highly symmetric structure for the $n=6$ cluster and closure of the first solvation shell at $n=12$. () 2006 American Institute of Physics. [DOI: 10.1063/1.2351675]

Multiply-charged anions (MCA's) and their hydrates are fundamental to condensed phase chemistry and biology in very diverse environments. For example, hydrated sulfate dianions play a key role in the homogeneous nucleation of ice particles by sulfate aerosols in the upper troposphere, ${ }^{1}$ activity lowering in fuel cells by adsorbed sulfate ions on platinum-based electrodes, ${ }^{2}$ and regulation of many metabolic and cellular processes. ${ }^{3}$ A microscopic, molecular level understanding of the role of hydrated MCA's in these and other processes requires elucidation of the solute-solvent interactions. Gas phase studies of hydrated MCA's can be particularly valuable in this regard, as they can probe the precise arrangement of the water molecules comprising the solvation shell and reveal novel hydrogen-bonding motifs differing from those seen in the hydration of singly-charged anions. ${ }^{4,5}$

In this paper, we use infrared (IR) spectroscopy to probe MCA hydration, focusing on clusters in which the sulfate dianion is solvated by a known number of water molecules. The results reported here represent the first IR spectra of MCA's in the gas phase. IR spectra of mass-selected $\mathrm{SO}_{4}^{2-} \cdot\left(\mathrm{H}_{2} \mathrm{O}\right)_{n}$ dianions $(n=3-24)$ were measured from $540-1850 \mathrm{~cm}^{-1}$, the spectral region associated with stretch-

\footnotetext{
${ }^{a)}$ Electronic mail: dneumark@berkeley.edu

${ }^{b)}$ Electronic mail: asmis@fhi-berlin.mpg.de
}

ing and bending modes of the sulfate core as well as characteristic intra- and intermolecular water modes. We find that the triply degenerate antisymmetric stretch of $\mathrm{SO}_{4}^{2-}$ provides a sensitive probe of the local solvation environment, as the degeneracy is lifted when the solvent molecules are arranged asymmetrically around the dianion core. Comparison with electronic structure calculations reveals substantial sizedependent evolution of the hydrogen-bonding in these clusters, providing direct spectroscopic evidence for a transition in binding motif at $n=6$ and solvent-shell closure at $n=12$.

MCA's are challenging to produce in the gas phase, owing to the Coulombic repulsion between the excess electrons. ${ }^{6}$ Most of the smaller dianions, including bare $\mathrm{SO}_{4}^{2-}$, are metastable, ${ }^{7}$ because the superposition of the longrange Coulomb repulsion and short-range electron binding creates a repulsive Coulomb barrier with respect to breakup to lower energy, singly-charged products. ${ }^{8}$ In solution and crystalline phases, MCA's are stabilized through electrostatic interactions with solvent molecules and counterions. In the gas phase, stabilization can be achieved by hydration, and both experiment and theory agree that a minimum of three water molecules are needed to stabilize the $\mathrm{SO}_{4}^{2-}$ dianion against spontaneous electron loss. ${ }^{9}$

Hydrated sulfate clusters were first observed in the gas phase by Blades and Kebarle. ${ }^{10}$ The first spectroscopic stud- 
ies on $\mathrm{SO}_{4}^{2-} \cdot\left(\mathrm{H}_{2} \mathrm{O}\right)_{n}$ clusters were performed by Wang and co-workers, ${ }^{9,11}$ whose photoelectron (PE) spectra provided an estimate of the repulsive Coulomb barrier and the stepwise hydration energies. Blackbody infrared radiative dissociation (BIRD) experiments ${ }^{12}$ yielded water loss rates for clusters with $n=3-17$ that implied the $n=6$ and $n=12 \mathrm{com}$ plexes are particularly stable, although subsequent experiments by Blades and Kebarle ${ }^{13}$ did not indicate enhanced stability for the $n=12$ complex. Several groups ${ }^{9,12,14-17}$ have investigated these clusters using electronic structure and molecular dynamics calculations, finding that the dianion is internally solvated, in contrast to many singly-charged anions. ${ }^{5}$ However, the computational studies yielded multiple structural isomers for each cluster size, each with varying degrees of water-water and water-sulfate hydrogen bonding, and the energetic orderings of the isomers for a given cluster size changed with the level of theory. Clearly, a direct experimental probe of the structure of these hydrated clusters is warranted.

Experiments were carried out on a tandem mass spectrometer-ion trap system ${ }^{18}$ using radiation from the broadly tunable Free Electron Laser for Infrared eXperiments, FELIX. ${ }^{19} \mathrm{SO}_{4}^{2-} \cdot\left(\mathrm{H}_{2} \mathrm{O}\right)_{n}$ ions were produced from a Perkin Elmer SCIEX atmospheric ion spray source using a $10^{-3} \mathrm{M}$ solution of tetrabutylammonium sulfate in water/ acetonitrile (15/85 volume ratio). An important experimental detail is that the mass-selected ions were cooled inside the ion trap via collisions with $\mathrm{He}$ gas at $17 \mathrm{~K}$ and 0.08 mbar. This cooling minimized the contribution of vibrational hot bands and energetically low-lying isomers to the infrared spectra. The trapped ions were irradiated by a single FEL macropulse (10-40 mJ energy and $5 \mu$ s duration), with a bandwidth of $\sim 2 \%$ of the central frequency. Resonant absorption of the FELIX radiation resulted in infrared multiphoton dissociation (IRMPD) of the parent ion; ${ }^{20}$ fragmentation was observed by extracting and mass-selecting the ions in the trap and measuring depletion of the parent ion signal. ${ }^{18}$

To aid interpretation of the spectra, electronic structure calculations with GAUSSIAN ${ }^{21}$ were performed to obtain vibrational spectra and optimized structures for selected clusters. For the smaller $(n=3-7)$ clusters, calculations were carried out at both the MP2 and DFT/B3LYP levels of theory, while only DFT was used for the larger $n=12$ and $n=13$ clusters. The TZVP+ basis set was the same one used by Wang et al. ${ }^{9}$

Experimental spectra for the various clusters are displayed in Fig. 1. Four bands, labeled A-D, that appear prominently in the spectra. These bands can be assigned by comparison with known features in the IR-spectra of the cluster constituents. In solution, the tetrahedrally symmetric sulfate dianion has two infrared-active modes: the antisymmetric stretching $\left(\nu_{3}\right)$ and bending $\left(\nu_{4}\right)$ modes at 1106 and $617 \mathrm{~cm}^{-1}$, respectively. ${ }^{22}$ These modes are assigned to bands B and D in Fig. 1, which fall in the ranges $1080-1110 \mathrm{~cm}^{-1}$ and $615-626 \mathrm{~cm}^{-1}$, respectively. The IR spectrum of liquid water also exhibits two bands in this spectral region: the intramolecular bending mode at $1645 \mathrm{~cm}^{-1},{ }^{23}$ and the intermolecular frustrated rotation, or librational mode, at $683 \mathrm{~cm}^{-1} .{ }^{24}$ Accordingly, these modes are assigned to bands

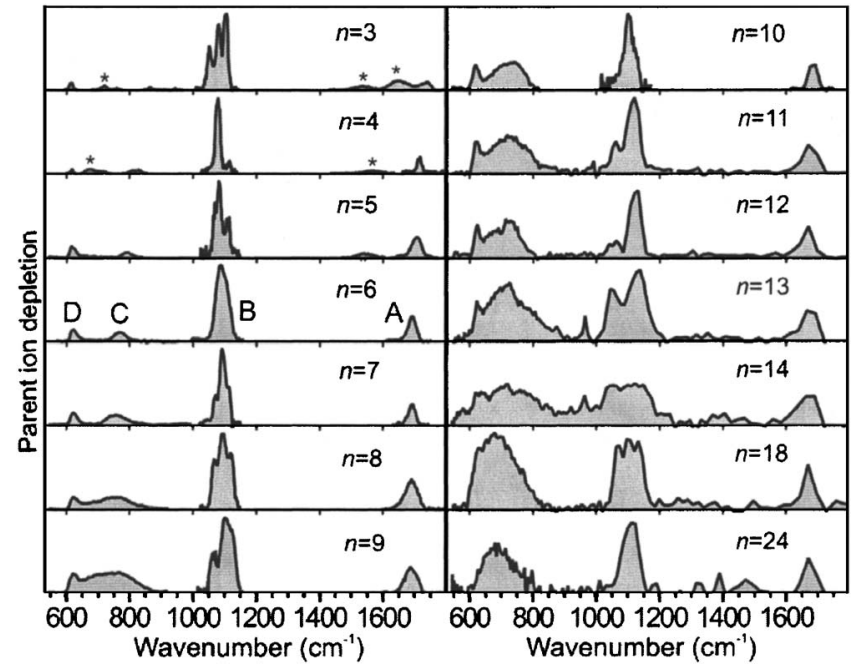

FIG. 1. Experimental spectra of $\mathrm{SO}_{4}^{2-} \cdot\left(\mathrm{H}_{2} \mathrm{O}\right)_{n}$ with $n=3-14,18$, and 24 . Bands A-D (indicated in the $n=6$ spectrum) are assigned as described in the text. Peaks indicated with an asterisk (*) are tentatively assigned to combination bands. For each cluster size, overview spectra were first acquired to pinpoint the bands, and then each band was scanned with the required FEL attenuations to avoid saturation of the absorption. This figure shows only the attenuated spectra.

A and C, which occur between 1735-1674 and $864-681 \mathrm{~cm}^{-1}$, respectively, in the IRMPD spectra. Several weaker bands in the spectra of the smallest clusters (indicated with ${ }^{*}$ in Fig. 1) are tentatively assigned to combination bands, based on results calculated by Gerber and Miller. ${ }^{25}$ Additional features in the spectra of $n=13$ and 14 clusters are attributed to changes in the hydration structure around the sulfate as discussed below.

The size-dependent trends observed in the experimental spectra provide further support for the assignments given above. For example, the water bands $\mathrm{A}$ and $\mathrm{C}$ are initially quite weak compared to the sulfate band $\mathrm{B}$, but grow with increasing cluster size. In addition, the band positions start out perturbed from the condensed phase vibrational origins, and then shift toward them gradually with increasing cluster size. Another notable size-dependent effect is the evolving shape of band $\mathrm{B}$, which is, for example, a triplet in the $n$ $=3,7$, and 8 clusters, a doublet in $n=5$ and 9 , and a singlet for $n=4$ and 6 . The $\nu_{3}$ mode assigned to this band is triply degenerate for a free sulfate ion with $T_{d}$ symmetry, so the shape of this band provides a sensitive probe of the local solvent environment. We also note that the width of the librational band $\mathrm{C}$ increases markedly above $n=6$, practically doubling from $n=6$ to 7 .

Further elucidation of the hydration configurations responsible for the experimental spectra is gained from electronic structure calculations. Figure 2 compares calculated (MP2/TZVP+) and experimental spectra for the smaller clusters $(n=3-6)$, along with images of the lowest energy cluster structures. The splitting pattern of band B provides a key point of comparison. The calculated spectra show that band B is quite strongly split for the low-symmetry $C_{2} n=3$ structure, where the solvation of the sulfate $\mathrm{O}$-atoms is anisotropic. This splitting disappears for the more isotropically solvated, highly symmetric $D_{2 d} n=4$ and $T_{d} n=6$ structures, 


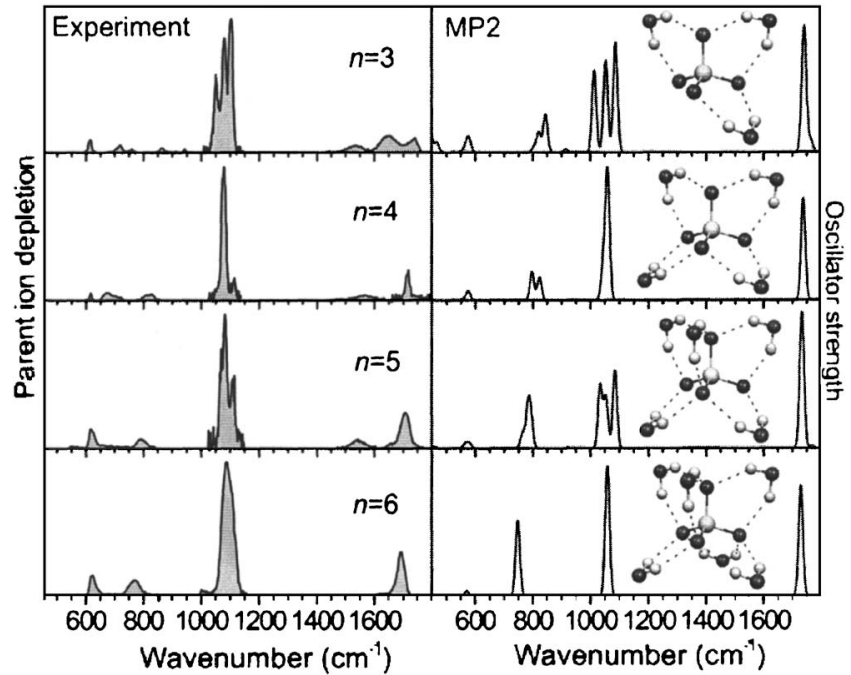

FIG. 2. Experimental vs calculated spectra for small sulfate-water clusters $(n=3-6)$. Calculated (MP2) spectra for the lowest energy isomers of the $n=3-6$ clusters are shown in the right-hand column $\left(15 \mathrm{~cm}^{-1}\right.$ FWHM Gaussian convolution), along with diagrams of the calculated cluster structures. The left-hand column shows the corresponding experimental spectra for comparison.

where each of the sulfate $\mathrm{O}$-atoms accept 2 and 3 equivalent hydrogen bonds, respectively. The $C_{2 \nu}$-symmetry $n=5$ structure is an intermediate case, exhibiting a smaller splitting than $n=3$, corresponding to a less anisotropic solvation environment. For all cases in Fig. 2, the calculated and experimental splitting patterns of band B are in reasonable agreement.

The water ligands in all of the structures in Fig. 2 exhibit the same "bridging" H-bonding motif, where each water acts as a double-donor to two sulfate $\mathrm{O}$-atoms. This result differs from other computational studies using DFT and/or smaller basis-sets, in which the lowest-energy structures included a mixture of bridging waters and waters with one sulfate and one water-water H-bond. ${ }^{9,15-17}$ For $n=3-6$, we found that the mixed structures have, in general, slightly higher energies (including zero-point vibrational correction) at the MP2/TZVP+ level of theory, and that their calculated spectra yield poorer matches with experiment. For example, Fig. 3(a) compares the structures and spectra for two isomers of $\mathrm{SO}_{4}^{2-}\left(\mathrm{H}_{2} \mathrm{O}\right)_{6}$ : the $T_{d}$ structure mentioned above, and a $C_{3}$ symmetry isomer where three of the water ligands form a cyclic trimer unit. The water-water H-bonds in the latter structure give rise to an extra band at $865 \mathrm{~cm}^{-1}$ in the calculated spectrum that is clearly absent from the experimental spectrum.

The structures in Fig. 2 imply that the driving force stabilizing these smaller clusters is solvation of the negativelycharged sulfate $\mathrm{O}$-atoms through hydrogen bonding. Following this trend, a seventh water cannot bind to the sulfate in the $n=6$ cluster without causing rearrangement of the initial six ligands. Figure 3(b) compares the calculated spectra for such a "rearranged" isomer to a " $6+1$ " isomer where the $n$ $=6 T_{d}$ core is retained, and the additional water is added to an outer shell. The latter isomer gives a better overall match to the IRMPD spectrum, particularly in the position of the intermolecular librational band that is expected to be most

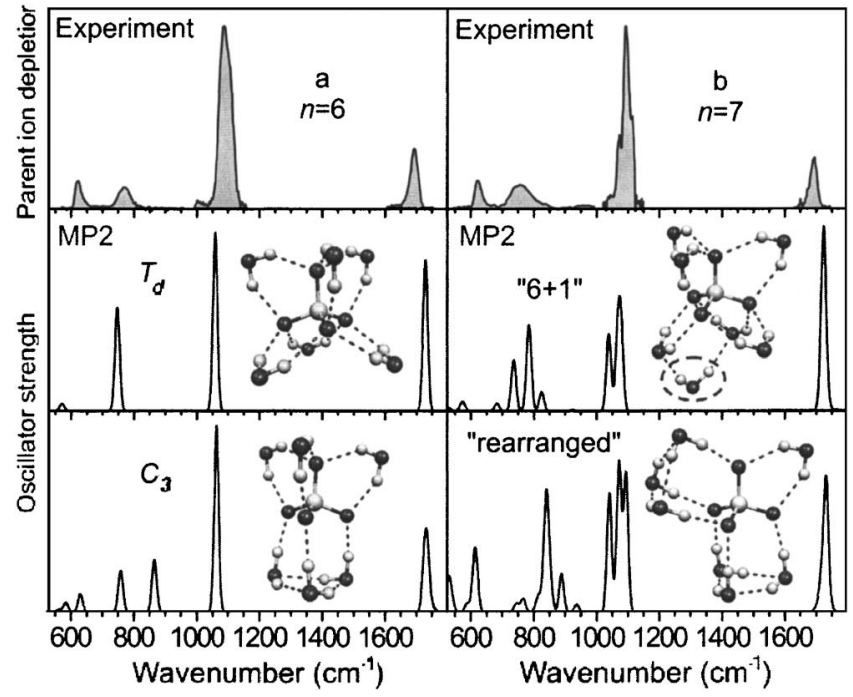

FIG. 3. (a) Calculated spectra and structures for $C_{3}$ and $T_{d}$ isomers of $n$ $=6$ clusters, compared with experimental spectrum. The computed relative energies indicate these $n=6$ isomers are essentially isoenergetic, with B3LYP favoring the $C_{3}$ isomer by $\sim 5 \mathrm{~kJ} / \mathrm{mol}$, and MP2 favoring the $T_{d}$ isomer by $\sim 1 \mathrm{~kJ} / \mathrm{mol}$. (b) Calculated spectra and structures for isomers of "rearranged" and " $6+1$ " isomers of $n=7$ clusters, compared with experimental spectrum. The dashed circle indicates the "outer shell" water in the " $6+1$ " structure (see text). Similar to $n=6$, the computed relative energies indicate these $n=7$ isomers are essentially isoenergetic, with both B3LYP and MP2 favoring the rearranged isomer by $\sim 5$ and $\sim 0.1 \mathrm{~kJ} / \mathrm{mol}$, respectively. All of the other H-bonding motifs involving water-water H-bonds produced $n=6$ and $n=7$ structural isomers that were significantly higher in energy.

sensitive to H-bond rearrangements. However, the two calculated spectra are not sufficiently distinct to rule out the presence of the rearranged isomer, so it may be that both isomers are represented in the IRMPD spectrum. For both $n=6$ and 7, the computed energies of two isomers are the same within the (estimated) uncertainty.

Figure 4 compares experimental and calculated spectra for the $n=12$ and $n=13$ clusters, along with the lowest energy calculated structures. The symmetric ( $T$ point group) $n=12$ structure is similar to those reported previously, ${ }^{15,17}$ and gives reasonable agreement with the experimental spectrum. In reference to the above discussion of the driving forces determining $\mathrm{H}$-bonding, this structure maximizes cluster stabilization by having each sulfate O-atom solvated by three H-bonds, and the waters organized into four cyclic trimer moieties, one for each face of the sulfate tetrahedron. The experimental $n=13$ spectrum shows a new peak near $950 \mathrm{~cm}^{-1}$, an otherwise inactive region in the spectra. It also shows a broadening of the librational band (band C) on the high-wave number side, and a shoulder on the low-wave number side of the sulfate stretching band (band B). The calculated spectrum exhibits similar features, most notably the peak in the $900 \mathrm{~cm}^{-1}$ region that is not present in the $n$ $=12$ spectrum. Examination of the calculated vibrational normal modes indicates that this mode is largely localized on the 13th water molecule, which resides outside the first solvation shell (dotted circle in Fig. 4). The appearance of this peak in both the experimental and calculated spectra provides strong indication that the 13th water does indeed go on the second solvation shell. Hence, comparison of experiment and theory 


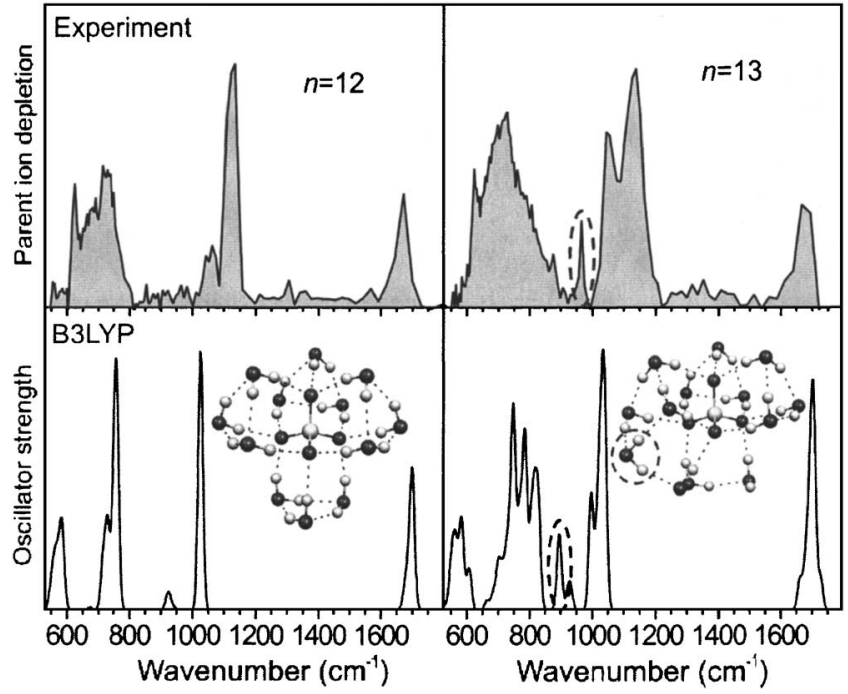

FIG. 4. Experimental vs calculated spectra for large sulfate-water clusters $(n=12,13)$. Calculated spectra (B3LYP) for the lowest energy isomers of the $n=12,13$ clusters are shown on the bottom $\left(15 \mathrm{~cm}^{-1}\right.$ FWHM Gaussian convolution), along with diagrams of the calculated cluster structures. The corresponding experimental spectra are shown above for comparison. The dashed circles for $n=13$ indicate the water in the 2 nd solvation shell, along with the associated vibrational bands in the calculated and experimental spectra. The B3LYP frequencies are not scaled. Comparative calculations for the smaller clusters have shown that B 3 LYP systematically underestimates the sulfate antisymmetric stretch frequencies by $\sim 80 \mathrm{~cm}^{-1}$, compared to the experimental data. However both B3LYP and MP2 reproduced the positions of the water bands quite well.

provides direct evidence for the genesis of a second solvation shell at $n=13$.

A picture of the sequential solvation of the sulfate dianion arises from the above discussion. For the smaller sizes $(n=3-6)$, each water adds to an open edge of the sulfate tetrahedron in a "bridging" fashion, forming H-bonds to adjacent sulfate O-atoms. This solvation structure can incorporate a maximum of six bridging waters. Above $n=6$, formation of water-water H-bonds becomes competitive with bridging ligands. This trend is made apparent by the broadening of the librational band for $n \geq 7$ and from comparison with theory (i.e., Fig. 3). The exact nature of the water solvation shell for $n=7$ cannot be determined, since there is no single spectroscopic feature that allows one to choose between the two calculated structures in Fig. 3. On the other hand, shell-closure at $n=12$ is indicated experimentally by the appearance of a new band in the spectra of the $n=13$ and $n=14$ clusters, associated with the libration of water molecules in the second solvation shell.

These results are the first infrared spectra of solvated MCA's in the gas phase. They provide new insights into the structure of these complexes. Overall, from the observed vibrational frequencies of both the dianion and the solvent, the complexes can be seen as a distorted tetrahedral $\mathrm{SO}_{4}^{2-}$ core surrounded by $\mathrm{H}_{2} \mathrm{O}$ molecules. We do not observe evidence for low-frequency $\left(\sim 1000 \mathrm{~cm}^{-1}\right)$ stretch and bend frequencies associated with a shared proton, in contrast to singlycharged cations and anions with strong hydrogen bonds such as $\mathrm{H}_{5} \mathrm{O}_{2}^{+}$and $\mathrm{H}_{3} \mathrm{O}_{2}^{-}{ }^{26,27}$ The absence of such features reflects the delocalization of negative charge in the sulfate ion over the four oxygen atoms, so that the individual hydrogen bonds with the surrounding water molecules are weaker than in singly-charged species with strong H-bonds.

This work is supported by the Air Force Office of Scientific Research under Grant No. F49550-06-1-0085, and by the German Research Foundation (DFG) as part of the Research Training Group \#788 and the Collaborative Research Center \#546. We gratefully acknowledge the support of the "Stichting voor Fundamenteel Onderzoek der Materie (FOM)" in providing the required beam time on FELIX and highly appreciate the skillful assistance of the FELIX staff. We thank B. Gao and Z. Liu for sharing unpublished details of their previous computational study of the $n=3-7$ complexes. ${ }^{16}$ We would also like to thank Y. Miller and R. B. Gerber for sharing unpublished anharmonic frequencies for the $n=3$ cluster.

${ }^{1}$ V. Ramanathan, P. J. Crutzen, J. T. Kiehl, and D. Rosenfeld, Science 294, 2119 (2001).

${ }^{2}$ N. Hoshi, M. Kuroda, T. Ogawa, O. Koga, and Y. Hori, Langmuir 20, 5066 (2004).

${ }^{3}$ A. Lee, P. A. Dawson, and D. Markovich, Int. J. Biochem. Cell Biol. 37, 1350 (2005)

${ }^{4}$ X. B. Wang, X. Yang, and L. S. Wang, Int. Rev. Phys. Chem. 21, 473 (2002).

${ }^{5}$ W. H. Robertson and M. A. Johnson, Annu. Rev. Phys. Chem. 54, 173 (2003).

${ }^{6}$ A. Dreuw and L. S. Cederbaum, Chem. Rev. 102, 181 (2002).

${ }^{7}$ A. Whitehead, R. Barrios, and J. Simons, J. Chem. Phys. 116, 2848 (2002).

${ }^{8}$ P. Skurski, J. Simons, X. B. Wang, and L. S. Wang, J. Am. Chem. Soc. 122, 4499 (2000).

${ }^{9}$ X. B. Wang, J. B. Nicholas, and L. S. Wang, J. Chem. Phys. 113, 10837 (2000).

${ }^{10}$ A. T. Blades and P. Kebarle, J. Am. Chem. Soc. 116, 10761 (1994).

${ }^{11}$ X. B. Wang, X. Yang, J. B. Nicholas, and L. S. Wang, Science 294, 1322 (2001).

${ }^{12}$ R. L. Wong and E. R. Williams, J. Phys. Chem. A 107, 10976 (2003).

${ }^{13}$ A. T. Blades and P. Kebarle, J. Phys. Chem. A 109, 8293 (2005).

${ }^{14}$ P. Jungwirth, J. E. Curtis, and D. J. Tobias, Chem. Phys. Lett. 367, 704 (2003).

${ }^{15}$ C. G. Zhan, F. Zheng, and D. A. Dixon, J. Chem. Phys. 119, 781 (2003).

${ }^{16}$ B. Gao and Z. Liu, J. Chem. Phys. 123, 224302 (2005).

${ }^{17}$ B. Gao and Z. F. Liu, J. Chem. Phys. 121, 8299 (2004).

${ }^{18}$ K. R. Asmis, M. Brummer, C. Kaposta, G. Santambrogio, G. von Helden, G. Meijer, K. Rademann, and L. Woste, Phys. Chem. Chem. Phys. 4, 1101 (2002)

${ }^{19}$ D. Oepts, A. F. G. van der Meer, and P. W. van Amersfoort, Infrared Phys. Technol. 36, 297 (1995).

${ }^{20}$ J. Oomens, A. J. A. van Roij, G. Meijer, and G. von Helden, Astrophys. J. 542, 404 (2000).

${ }^{21}$ M. J. Frisch, G. W. Trucks, H. B. Schlegel et al., GAUSSIAN 03, Gaussian, Inc., Wallingford, CT, 2004.

${ }^{22} \mathrm{~K}$. Nakamoto, Infrared and Raman Spectra of Inorganic and Coordination Compounds, 5th ed. (Wiley, New York, 1997).

${ }^{23}$ A. N. Rusk, D. Williams, and M. R. Querry, J. Opt. Soc. Am. 61, 895 (1971).

${ }^{24}$ H. R. Zelsmann, J. Mol. Struct. 350, 95 (1995).

${ }^{25}$ Y. Miller and R. G. Gerber (private communication).

${ }^{26}$ K. R. Asmis, N. L. Pivonka, G. Santambrogio, M. Brummer, C. Kaposta, D. M. Neumark, and L. Woste, Science 299, 1375 (2003).

${ }^{27}$ E. G. Diken, J. M. Headrick, J. R. Roscioli, J. C. Bopp, M. A. Johnson, and A. B. McCoy, J. Phys. Chem. A 109, 1487 (2005). 